\title{
Emptiness Formation Probability and Quantum Knizhnik-Zamolodchikov Equation
}

\author{
H. E. Boos 1 \\ Max-Planck Institut für Mathematik \\ Vivatsgasse 7, 53111 Bonn, Germany \\ V. E. Korepin \\ C.N. Yang Institute for Theoretical Physics \\ State University of New York at Stony Brook \\ Stony Brook, NY 11794-3840, USA \\ F.A. Smirnov \\ LPTHE, Tour 16, 1-er étage, 4, pl. Jussieu \\ 75252, Paris Cedex 05, France
}

\begin{abstract}
We consider the one-dimensional XXX spin 1/2 Heisenberg antiferromagnet at zero temperature and zero magnetic field. We are interested in a probability of formation of a ferromagnetic string $P(n)$ in the antiferromagnetic ground-state. We call it emptiness formation probability [EFP]. We suggest a new technique for computation of the EFP in the inhomogeneous case. It is based on the quantum Knizhnik-Zamolodchikov equation [qKZ]. We calculate EFP for $n \leq 6$ for inhomogeneous case. The homogeneous limit confirms our hypothesis about the relation of quantum correlations and number theory. We also make a conjecture about a structure of EFP for arbitrary $n$.
\end{abstract}

\footnotetext{
${ }^{1}$ on leave of absence from the Institute for High Energy Physics, Protvino, 142284, Russia

${ }^{2}$ Membre du CNRS
} 


\section{Introduction}

The Hamiltonian of the XXX Heisenberg spin chain can be written like this

$$
H=\sum_{i=-N}^{N}\left(\sigma_{i}^{x} \sigma_{i+1}^{x}+\sigma_{i}^{y} \sigma_{i+1}^{y}+\sigma_{i}^{z} \sigma_{i+1}^{z}-1\right)
$$

Here $2 N+1$ is the length of the lattice and $\sigma_{i}^{x}, \sigma_{i}^{y}, \sigma_{i}^{z}$ are Pauli matrices. We consider thermodynamic limit [ $N$ goes to infinity]. The sign in front of the Hamiltonian indicates that we are dealing with the antiferromagnetic case. We also imply periodic boundary conditions. The model was solved by Bethe in 1931, see [1]. The ground state was constructed by by Hulthén, see [2]. We shall denote the ground state in the thermodynamic limit by $|\mathrm{GS}\rangle$. The emptiness formation probability (EFP) for the XXX model is defined as follows:

$$
P(n)=\left\langle\mathrm{GS}\left|\prod_{j=1}^{n} P_{j}\right| \mathrm{GS}\right\rangle
$$

here $P_{j}=S_{j}^{z}+\frac{1}{2}$ is the projector on the state with the spin up in the $j$-th lattice site. The integer $n$ has a meaning of a length of a continuous ferromagnetic string. $P(n)$ is a probability that this string can appear in the antiferromagnetic ground-state. The importance of EFP was emphasized in [3].

$P(n)$ can be represented as $n$-multiple integral. The integral representation follows from the work of RIMS group. RIMS approach is based on vertex operators and bosonic representation of infinite-dimensional quantum algebras, see [4. 5]. The explicit formula for $P(n)$ in XXX limit was obtained in [7]:

$$
P(n)=\prod_{j=1}^{n} \int_{C} \frac{d \lambda_{j}}{2 \pi i} U_{n}\left(\lambda_{1}, \ldots, \lambda_{n}\right) T_{n}\left(\lambda_{1}, \ldots, \lambda_{n}\right)
$$

where

$$
U_{n}\left(\lambda_{1}, \ldots, \lambda_{n}\right)=\pi^{\frac{n(n+1)}{2}} \frac{\prod_{1 \leq k<j \leq n} \sinh \pi\left(\lambda_{j}-\lambda_{k}\right)}{\prod_{j=1}^{n} \sinh ^{n} \pi \lambda_{j}}
$$

and

$$
T_{n}\left(\lambda_{1}, \ldots, \lambda_{n}\right)=\frac{\prod_{j=1}^{n} \lambda_{j}^{j-1}\left(\lambda_{j}+i\right)^{n-j}}{\prod_{1 \leq k<j \leq n}\left(\lambda_{j}-\lambda_{k}-i\right)}
$$

The contour $C$ goes parallel to the real axis with the imaginary part confined between 0 and $-i$ for each integral.

In papers [8, 9, 10] we evaluated the integrals for $n \leq 5$. We discovered that these $P(n)$ can be expressed in terms of the values of Riemann zeta function at odd arguments, $\log 2$ and rational coefficients. We conjectured that this is a general property for all $P(n)$. In this paper we proved the property for $P(6)$. We think that all correlation functions

$$
\left\langle\mathrm{GS}\left|\sigma_{i_{1}}^{z} \sigma_{i_{2}}^{z} \ldots \sigma_{i_{m}}^{z}\right| \mathrm{GS}\right\rangle
$$

also have this property. Asymptotic behavior of $P(n)$ for large $n$ was studied in the papers [8], [10], [13] and [14]. The technique of calculation of these integrals, described in the paper [9] worked for $n=2,3,4$. In the paper [10] we calculated $P(5)$ by means of this technique. However, these 
computations are so complicated that it is problematic to generalize them to the case $n=6$. So we start looking for indirect methods of evaluation of the integrals (1.31).

It appeared to be useful to consider inhomogeneous case. In this case there are more free parameters. We call them inhomogeneity parameters and denote by $z_{1}, \ldots, z_{n}$. The EFP in the inhomogeneous case we shall denote by $P_{n}\left(z_{1}, \ldots, z_{n}\right)$. Let us remind to the reader that inhomogeneous models were used for evaluation of correlation functions from the very beginning. For the massive regime of the XXZ model the vertex operator approach was developed in [4, 5]. It allowed to express the correlation functions in terms of the trace functions. Special combinations of these trace functions satisfy the quantum Knizhnik-Zamolodchikov equation (qKZ), see [11, 12]. Later in paper [6] Miwa and Jimbo suggested that the correlation functions in the gap-less regime directly satisfy the qKZ equation. Since the XXX model belongs to the gap-less regime we shall use qKZ for evaluation of EFP in the inhomogeneous case. We suggest a general ansatz for $P_{n}\left(z_{1}, \ldots, z_{n}\right)$, see (3.20). This constitute a new method for computation of the EFP. On the other hand, it is easy to generalize the technique explained in [9] to the inhomogeneous case and calculate the EFP directly [for short ferromagnetic strings]. When we can compare results, they coincide.

The paper is organized as follows. In the Section 2 we discuss the relation of EFP to the qKZ and derive three important properties of the EFP in the inhomogeneous case. In Section 3 we apply a generalization of the technique described in [9] to the inhomogeneous case and compute $P_{n}$ directly for $n \leq 4$. Then we check that these $P_{n}$ satisfy all the properties, which follow from qKZ. This helps us to formulate a general ansatz for $P_{n}$ in the inhomogeneous case. Further, we suggest a new way of computing $P_{n}$. One can use the ansatz and general properties of $P_{n}$ [which follow from the qKZ]. In this way we get the explicit expressions for $P_{5}$ and $P_{6}$ in the inhomogeneous case. In Section 4 we discuss the homogeneous limit of $P(n)$ for $n \leq 6$. In particular, when $n \leq 5$ we reproduce our previous results, obtained in [8, 9, 10]. We also get the analytic expression for $P(6)$ in the homogeneous limit. Having this answer we can compare it with the numerical value for $P(6)$ obtained by the DMRG method in [10]. We also discuss the structure of EFP in the homogeneous case and offer some plausible conjectures. In the last Section 6 we discuss the results and outline some possible ways of a further progress.

\section{The EFP in the inhomogeneous case and the qKZ equa- tion.}

We believe that consideration of the inhomogeneous case instead of the homogeneous one can give us a new information about the EFP and other correlation functions.

A method of calculation of correlation functions, which we use was found in the papers [4], 5]. It is based on theory of infinite-dimensional quantized algebras and vertex operators. We shall need elements of this method. Let us introduce some notations.

We use the R-matrix:

$$
R(\lambda)=\frac{R_{0}(\lambda)}{\lambda+\pi i}\left(\begin{array}{cccc}
\lambda+\pi i & 0 & 0 & 0 \\
0 & -\lambda & \pi i & 0 \\
0 & \pi i & -\lambda & 0 \\
0 & 0 & 0 & \lambda+\pi i
\end{array}\right)
$$


where

$$
R_{0}(\lambda)=-\frac{\Gamma\left(\frac{\lambda}{2 \pi i}\right) \Gamma\left(\frac{1}{2}-\frac{\lambda}{2 \pi i}\right)}{\Gamma\left(-\frac{\lambda}{2 \pi i}\right) \Gamma\left(\frac{1}{2}+\frac{\lambda}{2 \pi i}\right)}
$$

notice that

$$
R_{0}(\lambda) R_{0}(-\lambda)=1
$$

This R-matrix appears in the rational limit from XXZ R-matrix, it is related to usual XXX R-matrix by

$$
R(\lambda)=\left(\sigma^{z} \otimes I\right) R_{X X X}(\lambda)\left(I \otimes \sigma^{z}\right)
$$

(similar transformation is needed when obtaining form factors of $S U(2)$-invariant Thirring model from SG ones [15]).

This R-matrix (2.11) satisfies the equation:

$$
R(-\pi i)=\left(\begin{array}{llll}
0 & 0 & 0 & 0 \\
0 & 1 & 1 & 0 \\
0 & 1 & 1 & 0 \\
0 & 0 & 0 & 0
\end{array}\right)
$$

Following [5] we introduce functions $g_{n}$ which satisfy the qKZ equations on level -4 [12]. We write the qKZ equations in their original form [15, 16] which takes into account explicitly symmetry:

$$
\begin{gathered}
g_{n}\left(\lambda_{1}, \cdots, \lambda_{j+1}, \lambda_{j}, \cdots, \lambda_{2 n}\right)_{\epsilon_{1}, \cdots, \epsilon_{j+1}^{\prime}, \epsilon_{j}^{\prime}, \cdots, \epsilon_{2 n}}= \\
=R\left(\lambda_{j}-\lambda_{j+1}\right)_{\epsilon_{j}^{\prime}, \epsilon_{j+1}^{\prime}}^{\epsilon_{j}, \epsilon_{j+1}} g_{n}\left(\lambda_{1}, \cdots, \lambda_{j}, \lambda_{j+1}, \cdots, \lambda_{2 n}\right)_{\epsilon_{1}, \cdots, \epsilon_{j}, \epsilon_{j+1}, \cdots, \epsilon_{2 n}} \\
g_{n}\left(\lambda_{1}, \cdots, \lambda_{2 n-1}, \lambda_{2 n}+2 \pi i\right)_{\epsilon_{1}, \cdots, \epsilon_{2 n-1}, \epsilon_{2 n}}=g_{n}\left(\lambda_{2 n}, \lambda_{1}, \cdots, \lambda_{2 n-1}\right)_{\epsilon_{2 n}, \epsilon_{1}, \cdots, \epsilon_{2 n-1}}
\end{gathered}
$$

Solutions to these equations are meromorphic functions with possible singularities at the points

$$
\Im\left(\lambda_{j}-\lambda_{k}\right)=\pi l, \quad l \in \mathbf{Z} \backslash 0
$$

For application to the correlation functions we are interested in a particular solution $g_{n}$ described in details in [4, 5]. Detailed study of this solution will be performed in a future publication, in the present paper we need only limited information about it. First, the $g_{n}$ is regular at $\Im\left(\lambda_{j}-\lambda_{k}\right)= \pm \pi$.

Moreover, much can be said about its values at these points [5] :

$$
\begin{gathered}
g_{n}\left(\lambda_{1}, \cdots, \lambda_{j-1}, \lambda_{j}, \lambda_{j}-\pi i, \lambda_{j+2}, \cdots, \lambda_{2 n}\right)_{\epsilon_{1}, \cdots, \epsilon_{j-1}, \epsilon_{j}, \epsilon_{j+1}, \epsilon_{j+2}, \cdots, \epsilon_{2 n}}= \\
=\delta_{\epsilon_{j},-\epsilon_{j+1}} g_{n-1}\left(\lambda_{1}, \cdots, \lambda_{j-1}, \lambda_{j+2}, \cdots, \lambda_{2 n}\right)_{\epsilon_{1}, \cdots, \epsilon_{j-1}, \epsilon_{j+2}, \cdots, \epsilon_{2 n}}
\end{gathered}
$$

Together with the symmetry (2.3), and (2.2) this equation implies

$$
\begin{gathered}
\sum_{\epsilon_{j}=-\epsilon_{j+1}} g_{n}\left(\lambda_{1}, \cdots, \lambda_{j-1}, \lambda_{j}, \lambda_{j}+\pi i, \lambda_{j+2}, \cdots, \lambda_{2 n}\right)_{\epsilon_{1}, \cdots, \epsilon_{j-1}, \epsilon_{j}, \epsilon_{j+1}, \epsilon_{j+2}, \cdots, \epsilon_{2 n}}= \\
=g_{n-1}\left(\lambda_{1}, \cdots, \lambda_{j-1}, \lambda_{j+2}, \cdots, \lambda_{2 n}\right)_{\epsilon_{1}, \cdots, \epsilon_{j-1}, \epsilon_{j+2}, \cdots, \epsilon_{2 n}}
\end{gathered}
$$

The emptiness formation probability $P_{n}$ is related to $g_{n}$ as follows

$$
P_{n}\left(z_{1}, \cdots, z_{n}\right)=g_{n}\left(\pi z_{1}, \cdots, \pi z_{n}, \pi\left(z_{n}+i\right), \cdots, \pi\left(z_{1}+i\right)\right)_{-, \cdots,-,+, \cdots,+}
$$


Now we want to establish some general properties of $P_{n}$ following from (2.3), (2.4), (2.5), (2.6). 1. Symmetry. The function $P_{n}\left(z_{1}, \cdots, z_{n}\right)$ is symmetric.

Proof. Obviously, it is enough to show that

$$
P_{n}\left(\cdots, z_{j}, z_{j+1}, \cdots\right)=P_{n}\left(\cdots, z_{j+1}, z_{j}, \cdots\right)
$$

This identity follows from (2.3) and from the fact that the R-matrix acts diagonally on the indices ,-- and,++ :

$$
\begin{gathered}
P_{n}\left(\cdots, z_{j}, z_{j+1}, \cdots\right)= \\
=g_{n}\left(\cdots, \pi z_{j}, \pi z_{j+1}, \cdots, \pi\left(z_{j+1}+i\right), \pi\left(z_{j}+i\right) \cdots\right)_{\ldots,-,-, \cdots,+,+, \cdots}= \\
=R_{0}\left(\pi\left(z_{j+1}-z_{j}\right)\right) R_{0}\left(\pi\left(z_{j}-z_{j+1}\right)\right) \\
\times g_{n}\left(\cdots, \pi z_{j+1}, \pi z_{j}, \cdots, \pi\left(z_{j}+i\right), \pi\left(z_{j+1}+i\right) \cdots\right)_{\ldots,-,-, \cdots,+,+, \cdots}= \\
=P_{n}\left(\cdots, z_{j+1}, z_{j}, \cdots\right)
\end{gathered}
$$

2. Vanishing. The function $P_{n}\left(z_{1}, \cdots, z_{n}\right)$ vanishes when $z_{k}=z_{j}+i$.

Proof. Due to the previous property it is sufficient to consider the case $k=1, j=2$. Let us put, first, $z_{1}=z+i, z_{2}=z^{\prime}$, then we shall take the limit $z \rightarrow z^{\prime}$.

$$
\begin{gathered}
P_{n}\left(z+i, z^{\prime}, \cdots, z_{n}\right)= \\
=g_{n}\left(\pi(z+i), \pi z^{\prime}, \cdots, \pi z_{n}, \pi\left(z_{n}+i\right), \cdots, \pi\left(z^{\prime}+i\right), \pi(z+2 i)\right)_{-,-, \cdots,-,+, \cdots,+,+}= \\
=g_{n}\left(\pi z, \pi(z+i), \pi z^{\prime}, \cdots, \pi z_{n}, \pi\left(z_{n}+i\right), \cdots, \pi\left(z^{\prime}+i\right),\right)_{+,-,-, \cdots,-,+, \cdots,+}
\end{gathered}
$$

where we used (2.1). Consider the limit $z \rightarrow z^{\prime}$. As it has been explained singularities do not occur for $\Im\left(\lambda_{j}-\lambda_{k}\right)= \pm \pi$. Moreover, the final result contains the fragment

$$
g_{n}(\cdots, \pi(z+i), \pi z \cdots) \ldots,-,-, \cdots
$$

which implies that the result vanishes due to (2.5). Because of absence of singularities this zero does not interfere with any pole, so

$$
P_{n}\left(z+i, z, \cdots, z_{n}\right)=0
$$

3. Normalization. The following asymptotic holds for $z_{1} \rightarrow \infty$ along the real axis:

$$
P_{n}\left(z_{1}, z_{2}, \cdots, z_{n}\right) \rightarrow \frac{1}{2} P_{n-1}\left(z_{2}, \cdots, z_{n}\right)
$$

Proof. One more property of the solution $g_{n}$ will be important for us. Using the integral formula from [5] one can show that $g_{n}\left(\lambda_{1}, \cdots, \lambda_{2 n-2}, \lambda, \lambda+\pi i\right)$ behaves as $O(1)$ when $\lambda \rightarrow \infty+i \kappa$ where $\kappa$ is a finite number. The leading term of asymptotic does not depend on $\kappa$. We shall use notation:

$$
g_{n}\left(\lambda_{1}, \cdots, \lambda_{2 n-2}, \lambda, \lambda+\pi i\right)_{\epsilon_{1}, \cdots, \epsilon_{2 n-2}, \epsilon_{2 n-1}, \epsilon_{2 n}} \rightarrow \widehat{g}_{n}\left(\lambda_{1}, \cdots, \lambda_{2 n-2}\right)_{\epsilon_{1}, \cdots, \epsilon_{2 n-2} ; \epsilon_{2 n-1}, \epsilon_{2 n}}
$$

The function $\widehat{g}_{n}$ possesses important property of symmetry with respect to last two indices because

$$
\begin{aligned}
& g_{n}\left(\lambda_{1}, \cdots, \lambda_{2 n-2}, \lambda, \lambda+\pi i\right)_{\epsilon_{1}, \cdots, \epsilon_{2 n-2}, \epsilon_{2 n-1}, \epsilon_{2 n}}= \\
= & g_{n}\left(\lambda-\pi i, \lambda_{1}, \cdots, \lambda_{2 n-2}, \lambda\right)_{\epsilon_{2 n} \epsilon_{1}, \cdots, \epsilon_{2 n-2}, \epsilon_{2 n-1}} \\
= & R\left(\lambda_{1}-\lambda+\pi i\right)_{\epsilon_{1}, \epsilon_{2 n}^{\prime}, \sigma_{2 n-3}} \cdots R\left(\lambda_{2 n-2}-\lambda+\pi i\right)_{\epsilon_{2 n-2}, \sigma_{1}}^{e_{2 n}} \\
\times & g_{n}\left(\lambda_{1}, \cdots, \lambda_{2 n-2}, \lambda-\pi i, \lambda\right)_{\epsilon_{1}^{\prime}, \cdots, \epsilon_{2 n-2}^{\prime}, \epsilon_{2 n}^{\prime}, \epsilon_{2 n-1}} \rightarrow \\
\rightarrow & \operatorname{sign}\left(\epsilon_{2 n-1}, \epsilon_{2 n}\right) \widehat{g}_{n}\left(\lambda_{1}, \cdots, \lambda_{2 n-2}\right)_{\epsilon_{1}, \cdots, \epsilon_{2 n-2} ; \epsilon_{2 n}, \epsilon_{2 n-1}}
\end{aligned}
$$


with the sign function

$$
\operatorname{sign}\left(\epsilon_{1}, \epsilon_{2}\right)= \begin{cases}-1 & \text { if } \epsilon_{1}=\epsilon_{2} \\ 1 & \text { if } \epsilon_{1}=-\epsilon_{2}\end{cases}
$$

where we used the asymptotic of the R-matrix

$$
R(\lambda) \underset{\lambda \rightarrow \infty}{\longrightarrow}(-i) \cdot \operatorname{diag}(1,-1,-1,1)
$$

From the equation (2.11) we conclude that

$$
\begin{gathered}
\widehat{g}_{n}\left(\lambda_{1}, \cdots, \lambda_{2 n-2}\right)_{\epsilon_{1}, \cdots, \epsilon_{2 n-2} ; \epsilon_{2 n-1}, \epsilon_{2 n}}=0 \quad \text { if } \epsilon_{2 n-1}=\epsilon_{2 n} \\
\widehat{g}_{n}\left(\lambda_{1}, \cdots, \lambda_{2 n-2}\right)_{\epsilon_{1}, \cdots, \epsilon_{2 n-2} ;+,-}=\widehat{g}_{n}\left(\lambda_{1}, \cdots, \lambda_{2 n-2}\right)_{\epsilon_{1}, \cdots, \epsilon_{2 n-2}} ;-,+
\end{gathered}
$$

The relation (2.13) allows to calculate $\widehat{g}_{n}\left(\lambda_{1}, \cdots, \lambda_{2 n-2}\right)_{\epsilon_{1}, \cdots, \epsilon_{2 n-2} ;+,-}$ :

$$
\begin{gathered}
g_{n-1}\left(\lambda_{1}, \cdots, \lambda_{2 n-2}\right)_{\epsilon_{1}, \cdots, \epsilon_{2 n-2}}= \\
=\widehat{g}_{n}\left(\lambda_{1}, \cdots, \lambda_{2 n-2}\right)_{\epsilon_{1}, \cdots, \epsilon_{2 n-2}} ;+,-+\widehat{g}_{n}\left(\lambda_{1}, \cdots, \lambda_{2 n-2}\right)_{\epsilon_{1}, \cdots, \epsilon_{2 n-2} ;-,+}= \\
=2 \widehat{g}_{n}\left(\lambda_{1}, \cdots, \lambda_{2 n-2}\right)_{\epsilon_{1}, \cdots, \epsilon_{2 n-2}} ;+,-
\end{gathered}
$$

Now the normalization (2.9) follows from

$$
\begin{gathered}
P_{n}\left(z_{1}, z_{2}, \cdots, z_{n}\right)= \\
=g_{n}\left(\pi z_{1}, \pi z_{2}, \cdots, \pi z_{n}, \pi\left(z_{n}+i\right), \cdots, \pi\left(z_{2}+i\right), \pi\left(z_{1}+i\right)\right)_{-,-, \cdots,-,+, \cdots,+,+}= \\
=g_{n}\left(\pi z_{2}, \cdots, \pi z_{n}, \pi\left(z_{n}+i\right), \cdots, \pi\left(z_{2}+i\right), \pi\left(z_{1}+i\right), \pi\left(z_{1}+2 i\right)\right)_{-, \cdots,-,+, \cdots,+,+,-} \\
\underset{z_{1 \rightarrow \infty}}{\longrightarrow} \widehat{g}_{n}\left(\pi z_{2}, \cdots, \pi z_{n}, \pi\left(z_{n}+i\right), \cdots, \pi\left(z_{2}+i\right)\right)_{-, \cdots,-,+, \cdots,+;},+,- \\
=\frac{1}{2} P_{n-1}\left(z_{2}, \cdots, z_{n}\right)
\end{gathered}
$$

where the formula (2.10) was used.

The above three properties will be very useful for further consideration.

QED

\section{Explicit expressions for EFP.}

In paper [6] Jimbo and Miwa suggested an integral representation as a solution to the qKZ (2.3) - (2. 国) which also satisfies the property (2.5). Using the relation (2.7) one gets the integral representation which is the direct generalization of the formula (1.3) to the inhomogeneous case

$$
\begin{gathered}
P_{n}\left(z_{1}, \ldots, z_{n}\right)=\pi^{\frac{n(n+1)}{2}} \prod_{k<j} \frac{\sinh \left(\pi\left(z_{k}-z_{j}\right)\right)}{\pi\left(z_{k}-z_{j}\right)} \int_{-i / 2-\infty}^{-i / 2+\infty} \frac{d \lambda_{1}}{2 \pi i} \ldots \int_{-i / 2-\infty}^{-i / 2+\infty} \frac{d \lambda_{n}}{2 \pi i} \prod_{k<j} \frac{\sinh \left(\pi\left(\lambda_{j}-\lambda_{k}\right)\right)}{\left(\lambda_{j}-\lambda_{k}-i\right)} . \\
\cdot \frac{\prod_{j=1}^{n}\left(\prod_{k=1}^{j-1}\left(\lambda_{j}-z_{k}-i\right) \prod_{k=j+1}^{n}\left(\lambda_{j}-z_{k}\right)\right)}{\prod_{j=1}^{n} \prod_{k=1}^{n} \sinh \left(\pi\left(\lambda_{j}-z_{k}\right)\right)}
\end{gathered}
$$

where for the moment we will consider the inhomogeneity parameters $z_{j}$ as distinct real numbers. These parameters also may be considered as arbitrary complex numbers, but the integration contours in this case should be taken in such a way that they separate the singularities of the integrand in the same manner as the contours in the above formula (3.四) for the real values $z_{j}$. 
For evaluation of the integral (3.14) we can use the technique described in [9]. We obtain for the first four values of $P_{n}\left(z_{1}, \ldots, z_{n}\right)$ :

$$
\begin{gathered}
P_{1}=\frac{1}{2} \\
P_{2}=\frac{1}{3}+A_{2,1}\left(z_{1}, z_{2}\right) G\left(z_{1}-z_{2}\right) \\
P_{3}=\frac{1}{4}+A_{3,1}\left(z_{1}, z_{2} \mid z_{3}\right) G\left(z_{1}-z_{2}\right)+A_{3,1}\left(z_{1}, z_{3} \mid z_{2}\right) G\left(z_{1}-z_{3}\right)+A_{3,1}\left(z_{2}, z_{3} \mid z_{1}\right) G\left(z_{2}-z_{3}\right) \\
P_{4}=\frac{1}{5}+A_{4,1}\left(z_{1}, z_{2} \mid z_{3}, z_{4}\right) G\left(z_{1}-z_{2}\right)+A_{4,1}\left(z_{1}, z_{3} \mid z_{2}, z_{4}\right) G\left(z_{1}-z_{3}\right)+A_{4,1}\left(z_{1}, z_{4} \mid z_{2}, z_{3}\right) G\left(z_{1}-z_{4}\right)+ \\
A_{4,1}\left(z_{2}, z_{3} \mid z_{1}, z_{4}\right) G\left(z_{2}-z_{3}\right)+A_{4,1}\left(z_{2}, z_{4} \mid z_{1}, z_{3}\right) G\left(z_{2}-z_{4}\right)+A_{4,1}\left(z_{3}, z_{4}, \mid z_{1}, z_{2}\right) G\left(z_{3}-z_{4}\right)+ \\
A_{4,2}\left(z_{1}, z_{2} \mid z_{3}, z_{4}\right) G\left(z_{1}-z_{2}\right) G\left(z_{3}-z_{4}\right)+A_{4,2}\left(z_{1}, z_{3} \mid z_{2}, z_{4}\right) G\left(z_{1}-z_{3}\right) G\left(z_{2}-z_{4}\right)+ \\
A_{4,2}\left(z_{1}, z_{4} \mid z_{2}, z_{3}\right) G\left(z_{1}-z_{4}\right) G\left(z_{2}-z_{3}\right)
\end{gathered}
$$

where

$$
\begin{gathered}
G(x)=\left(x^{2}+1\right)(\beta(i x)+\beta(-i x))=2 \sum_{k=1}^{\infty}(-1)^{k} \cdot k \cdot \frac{x^{2}+1}{x^{2}+k^{2}} \\
\beta(x)=\frac{1}{2}\left(\psi\left(\frac{1+x}{2}\right)-\psi\left(\frac{x}{2}\right)\right)=\sum_{k=0}^{\infty} \frac{(-1)^{k}}{x+k} \\
\psi(x)=\frac{d}{d x} \ln \Gamma(x)
\end{gathered}
$$

and the functions

$$
\begin{aligned}
A_{2,1}\left(z_{1}, z_{2}\right) & =\bar{Q}_{2,1}\left(z_{1}, z_{2}\right) \\
A_{3,1}\left(z_{1}, z_{2} \mid z_{3}\right) & =\frac{\bar{Q}_{3,1}\left(z_{1}, z_{2} \mid z_{3}\right)}{z_{13} z_{23}} \\
A_{4,2}\left(z_{1}, z_{2} \mid z_{3}, z_{4}\right) & =\frac{\bar{Q}_{4,2}\left(z_{1}, z_{2} \mid z_{3}, z_{4}\right)}{z_{13} z_{14} z_{23} z_{24}} \\
A_{4,1}\left(z_{1}, z_{2} \mid z_{3}, z_{4}\right) & =\frac{\bar{Q}_{4,1}\left(z_{1}, z_{2} \mid z_{3}, z_{4}\right)}{z_{13} z_{14} z_{23} z_{24}}
\end{aligned}
$$

with the polynomials

$$
\begin{gathered}
\bar{Q}_{2,1}\left(z_{1}, z_{2}\right)=\frac{1}{6} \\
\bar{Q}_{3,1}\left(z_{1}, z_{2} \mid z_{3}\right)=\frac{1}{12}\left(z_{13} z_{23}-1\right) \\
\bar{Q}_{4,2}\left(z_{1}, z_{2} \mid z_{3}, z_{4}\right)=\frac{1}{36}\left\{\left(z_{13} z_{24}-1\right)\left(z_{14} z_{23}-1\right)+\frac{2}{5}\left(z_{12}^{2}+\frac{3}{2}\right)\left(z_{34}^{2}+\frac{3}{2}\right)+\frac{3}{2}\right\} \\
\bar{Q}_{4,1}\left(z_{1}, z_{2} \mid z_{3}, z_{4}\right)=2 \bar{Q}_{4,2}\left(z_{1}, z_{2} \mid z_{3}, z_{4}\right)-\frac{1}{60}\left(z_{12}^{2}+4\right)\left(z_{34}^{2}+1\right)
\end{gathered}
$$


and by definition

$$
z_{j k}=z_{j}-z_{k}
$$

The function $G(x)(3.6)$ is actually real for real $x$ and even

$$
G(-x)=G(x)
$$

Besides, for small $x$ one has an expansion

$$
G(x)=-2\left(1+x^{2}\right) \sum_{k=0}^{\infty}(-1)^{k} x^{2 k} \zeta_{a}(2 k+1)
$$

with the alternating zeta series defined in (4.11). In particular,

$$
G(0)=-2 \ln 2
$$

The following limit is well defined

$$
G( \pm \infty)=-\frac{1}{2}
$$

We shall also need the following relation

$$
G(x-i)=\alpha(x)+\gamma(x) G(x)
$$

where

$$
\alpha(x)=-\frac{(x-2 i)}{(x-i)}, \quad \gamma(x)=-\frac{x(x-2 i)}{(x+i)(x-i)}
$$

In particular,

$$
G( \pm i)=-2
$$

Looking at the answers (3.2-3.5) we can conclude that they satisfy the general three properties from the previous section and one more property, namely, the translational invariance:

$$
P_{n}\left(z_{1}+a, \ldots, z_{n}+a\right)=P_{n}\left(z_{1}, \ldots, z_{n}\right)
$$

which actually follows from the integral representation (3.11). This property means that $P_{n}$ depends only on differences of $n$ parameters $z_{j}$.

One can also suggest a general ansatz:

$$
P_{n}\left(z_{1}, \ldots, z_{n}\right)=\sum_{l=0}^{\left[\frac{n}{2}\right]}\left\{A_{n, l}\left(z_{1}, z_{2}\left|z_{3}, z_{4}\right| \ldots\left|z_{2 l-1}, z_{2 l}\right| z_{2 l+1} \ldots z_{n}\right) \prod_{j=1}^{l} G\left(z_{2 j-1}-z_{2 j}\right)+\text { permutations }\right\}
$$

where $A_{n, l}\left(z_{1}, z_{2}\left|z_{3}, z_{4}\right| \ldots\left|z_{2 l-1}, z_{2 l}\right| z_{2 l+1} \ldots z_{n}\right)$ are some rational functions which depend only on differences $z_{j}-z_{k}$. We also imply that these functions are symmetric under independent transpositions $z_{1} \leftrightarrow z_{2}, z_{3} \leftrightarrow z_{4}, \ldots, z_{2 l-1} \leftrightarrow z_{2 l}$ and any permutation of the residual variables $z_{2 l+1} \ldots z_{n}$. Also we require a symmetry of this function under transposition of any pair $z_{2 r-1} z_{2 r} \leftrightarrow z_{2 s-1} z_{2 s}$ for $r, s \leq l$. It is implied that the permutations in $(3 \sqrt[20]{)})$ do not involve those sets of variables for which the function $A_{n, l}$ is already symmetric. We also take

$$
A_{n, 0}\left(z_{1}, \ldots, z_{n}\right)=\frac{1}{n+1}
$$


and 3

$$
A_{n, l}\left(z_{1}, z_{2}\left|z_{3}, z_{4}\right| \ldots\left|z_{2 l-1}, z_{2 l}\right| z_{2 l+1} \ldots z_{n}\right)=\frac{Q_{n, l}\left(z_{1}, z_{2}\left|z_{3}, z_{4}\right| \ldots\left|z_{2 l-1}, z_{2 l}\right| z_{2 l+1} \ldots z_{n}\right)}{\prod_{1 \leq j<k \leq n}\left(z_{j}-z_{k}\right)}
$$

where $Q_{n, l}$ are some polynomials of maximum power $n-1$ for each variable $z_{k}$. Moreover we expect that these polynomials have factorized form, namely,

$$
\begin{gathered}
Q_{n, l}\left(z_{1}, z_{2}\left|z_{3}, z_{4}\right| \ldots\left|z_{2 l-1}, z_{2 l}\right| z_{2 l+1} \ldots z_{n}\right)=\left(z_{1}-z_{2}\right)\left(z_{3}-z_{4}\right) \ldots\left(z_{2 l-1}-z_{2 l}\right) \prod_{2 l+1 \leq j<k \leq n}\left(z_{j}-z_{k}\right) . \\
\bar{Q}_{n, l}\left(z_{1}, z_{2}\left|z_{3}, z_{4}\right| \ldots\left|z_{2 l-1}, z_{2 l}\right| z_{2 l+1} \ldots z_{n}\right)
\end{gathered}
$$

where the polynomials $\bar{Q}_{n, l}\left(z_{1}, z_{2}\left|z_{3}, z_{4}\right| \ldots\left|z_{2 l-1}, z_{2 l}\right| z_{2 l+1} \ldots z_{n}\right)$ depend only on differences of variables $z_{j}-z_{k}$. Also they have the same symmetry properties as the function $A_{n, l}\left(z_{1}, z_{2}\left|z_{3}, z_{4}\right| \ldots\left|z_{2 l-1}, z_{2 l}\right| z_{2 l+1} \ldots z_{n}\right)$ described above. The maximum powers for each variable may be readily calculated from the corresponding power of the polynomial $Q_{n l}$.

Let us rewrite the properties 2 and 3 from the Section 2 in the following form

$$
\begin{gathered}
P_{n}\left(z_{1}, \ldots, z_{n-2}, z_{n-1}, z_{n-1} \pm i\right)=0 \\
\lim _{z_{n} \rightarrow \infty} P_{n}\left(z_{1}, \ldots, z_{n-1}, z_{n}\right)=\frac{1}{2} P_{n-1}\left(z_{1}, \ldots, z_{n-1}\right)
\end{gathered}
$$

As we shall see below together with our main ansatz (3.20) they completely fix the answer for $P_{5}\left(z_{1}, \ldots, z_{5}\right)$ and $P_{6}\left(z_{1}, \ldots, z_{6}\right)$. Therefore we may expect that it also happens for any $P_{n}\left(z_{1}, \ldots, z_{n}\right)$.

Let us start our consideration from the analysis of the relation (3.25). First let us make a simple observation that it has an obvious solution corresponding to the case when all the variables $z_{j}$ go to infinity 1 i.e.

$$
P_{n}(\infty, \ldots, \infty)=\frac{1}{2^{n}}
$$

Now we can analyze corollaries of the relation (3.25) and our general ansatz (3.20). It can be seen that this is equivalent to the following chain of recurrent relations for the functions $A_{n, l}$

$$
\begin{gathered}
A_{2 k+1, l}\left(z_{1}, z_{2}\left|z_{3}, z_{4}\right| \ldots\left|z_{2 l-1}, z_{2 l}\right| z_{2 l+1} \ldots z_{2 k}, \infty\right)- \\
\frac{1}{2}\left\{A_{2 k+1, l+1}\left(z_{1}, z_{2}\left|z_{3}, z_{4}\right| \ldots\left|\infty, z_{2 l+1}\right| z_{2 l+2}, \ldots, z_{2 k}\right)+\text { permutations }\right\}= \\
\frac{1}{2} A_{2 k, l}\left(z_{1}, z_{2}\left|z_{3}, z_{4}\right| \ldots \mid z_{2 l+1}, \ldots, z_{2 k}\right) \quad \text { for } \quad l=0,1, \ldots, k \\
A_{2 k, l}\left(z_{1}, z_{2}\left|z_{3}, z_{4}\right| \ldots\left|z_{2 l-1}, z_{2 l}\right| z_{2 l+1} \ldots z_{2 k-1}, \infty\right)- \\
\frac{1}{2}\left\{A_{2 k, l+1}\left(z_{1}, z_{2}\left|z_{3}, z_{4}\right| \ldots\left|\infty, z_{2 l+1}\right| z_{2 l+2}, \ldots, z_{2 k-1}\right)+\text { permutations }\right\}= \\
\frac{1}{2} A_{2 k-1, l}\left(z_{1}, z_{2}\left|z_{3}, z_{4}\right| \ldots \mid z_{2 l+1}, \ldots, z_{2 k-1}\right) \quad \text { for } \quad l=0,1, \ldots, k-1
\end{gathered}
$$

\footnotetext{
${ }^{3}$ Since $A_{n, 0}$ are just constants below we will not write their arguments any more.

${ }^{4}$ The limits $z_{n} \rightarrow \infty, \ldots, z_{1} \rightarrow \infty$ should be taken consequently
} 
where permutations are taken only for the variables $z_{2 l+1}, z_{2 l+2}, \ldots z_{2 k}$ for $(3.27)$ and $z_{2 l+1}, z_{2 l+2}, \ldots z_{2 k-1}$ for (3.28) in such a way that the variables within symmetric sets are not permuted. In the formula (3.27) it is also implied that

$$
A_{2 k+1, k+1}=0 \text {. }
$$

Up to the moment it is not completely clear how to write down in a closed form all the equations for the functions $A_{n, k}$ which follow from the relation (3.24).

Let us write them down together with the equations (3.27) and (3.28) consequently for $n=$ $2,3,4,5,6$.

The case $n=2$

Since $k=1$ in this case we have only one equation in the set (3.28)

$$
A_{2,0}-\frac{1}{2} A_{2,1}\left(\infty, z_{1}\right)=\frac{1}{2} A_{1,0}
$$

which is obvious because as it can be seen from eqs. (3.2-3.3), (3.7) and (3.8) all the functions $A$ here are just numbers, namely,

$$
\begin{gathered}
A_{1,0}=\frac{1}{2} \\
A_{2,0}=\frac{1}{3}, \quad A_{2,1}\left(z_{1}, z_{2}\right)=\frac{1}{6}
\end{gathered}
$$

The second property, namely, the formula (3.24) is also trivially satisfied because using eq. (3.17), (3.18) we have from (3.3)

$$
\begin{gathered}
P_{2}\left(z_{1}, z_{1}+i\right)=A_{2,0}+A_{2,1}\left(z_{1}, z_{1}+i\right) G(-i)= \\
A_{2,0}-2 A_{2,1}\left(z_{1}, z_{1}+i\right)=0
\end{gathered}
$$

So the equation corresponding to the second property is just the last relation in formula (3.31).

The case $n=3$

Since $n$ is odd the first property (3.25) corresponds to the relations (3.27) with $k=1$. Here we have two of them

$$
\begin{gathered}
A_{3,1}\left(z_{1}, z_{2} \mid \infty\right)=\frac{1}{2} A_{2,1}\left(z_{1}, z_{2}\right) \\
A_{3,0}\left(z_{1}, z_{2} \mid \infty\right)-\frac{1}{2}\left\{A_{3,1}\left(\infty, z_{1} \mid z_{2}\right)+A_{3,1}\left(\infty, z_{2} \mid z_{1}\right)\right\}=\frac{1}{2} A_{2,1}\left(z_{1}, z_{2}\right)
\end{gathered}
$$

The second property (3.24) i.e. the equation

$$
P_{3}\left(z_{1}, z_{2}, z_{2}+i\right)=0
$$

is equivalent to the following two relations

$$
\begin{gathered}
A_{3,1}\left(z_{1}, z_{2} \mid z_{2}+i\right)+\gamma\left(z_{12}\right) A_{3,1}\left(z_{1}, z_{2}+i \mid z_{2}\right)=0 \\
A_{3,0}+\alpha\left(z_{12}\right) A_{3,1}\left(z_{1}, z_{2}+i \mid z_{2}\right)-2 A_{3,1}\left(z_{2}, z_{2}+i \mid z_{1}\right)=0
\end{gathered}
$$


Substituting here $A_{3,1}$ defined in (3.7) and (3.9) $\left(A_{3,0}=1 / 4\right)$ one can easily check that these two equations are satisfied.

The case $n=4$

As in the previous case the first property (3.25) is equivalent to the set of two relations (see (3.28) for $k=2$ )

$$
\begin{gathered}
A_{4,1}\left(z_{1}, z_{2} \mid z_{3}, \infty\right)-\frac{1}{2} A_{4,2}\left(z_{1}, z_{2} \mid \infty, z_{3}\right)=\frac{1}{2} A_{3,1}\left(z_{1}, z_{2} \mid z_{3}\right) \\
A_{4,0}-\frac{1}{2}\left\{A_{4,1}\left(z_{1}, \infty \mid z_{2}, z_{3}\right)+A_{4,1}\left(z_{2}, \infty \mid z_{1}, z_{3}\right)+A_{4,1}\left(z_{3}, \infty \mid z_{1}, z_{2}\right)\right\}=\frac{1}{2} A_{3,0}
\end{gathered}
$$

The Property 2 (3.24) for $n=4$ is equivalent to the following set of equations

$$
\begin{gathered}
\gamma\left(z_{23}\right) A_{4,2}\left(z_{1}, z_{3} \mid z_{2}, z_{3}+i\right)+\gamma\left(z_{13}\right) A_{4,2}\left(z_{1}, z_{3}+i \mid z_{2}, z_{3}\right)=0 \\
A_{4,1}\left(z_{1}, z_{2} \mid z_{3}, z_{3}+i\right)-2 A_{4,2}\left(z_{1}, z_{2} \mid z_{3}, z_{3}+i\right)=0 \\
A_{4,1}\left(z_{1}, z_{3} \mid z_{2}, z_{3}+i\right)+\gamma\left(z_{13}\right) A_{4,1}\left(z_{1}, z_{3}+i \mid z_{2}, z_{3}\right)+\alpha\left(z_{23}\right) A_{4,2}\left(z_{1}, z_{3} \mid z_{2}, z_{3}+i\right)=0 \\
A_{4,0}+\alpha\left(z_{13}\right) A_{4,1}\left(z_{1}, z_{3}+i \mid z_{2}, z_{3}\right)+\alpha\left(z_{23}\right) A_{4,1}\left(z_{2}, z_{3}+i \mid z_{1}, z_{3}\right)-2 A_{4,1}\left(z_{3}, z_{3}+i \mid z_{1}, z_{2}\right)=0
\end{gathered}
$$

Again using eqs. (3.7) and (3.10-3.11) one can check that the equations (3.35-3.36) are satisfied.

So we have established that, in fact, the formulae (3.7) with the definitions (3.8-3.11) provide the solution to the equations (3.30) and (3.31) for $n=2$, eqs. (3.32) and (3.34) for $n=3$, eqs. (3.35) and (3.36) for $n=4$.

We may also go an opposite way, namely, demand all these equations, solve them in framework of the ansatz (3.20-3.23) and then come to the result for $P_{n}$. Let us mention that this way seems to be much easier than the direct calculation of the integral (3.11) as we did before.

For $n=5,6$ we would like to try this new way of getting the result for EFP.

The case $n=5$

First as above we write down consequences of the properties (3.25) and (3.24). The first one (3.25) is equivalent to the set of three relations which can be obtained from eqs. (3.27) by taking $k=2$

$$
\begin{gathered}
A_{5,2}\left(z_{1}, z_{2}\left|z_{3}, z_{4}\right| \infty\right)=\frac{1}{2} A_{4,2}\left(z_{1}, z_{2} \mid z_{3}, z_{4}\right) \\
A_{5,1}\left(z_{1}, z_{2} \mid z_{3}, z_{4}, \infty\right)-\frac{1}{2}\left\{A_{5,2}\left(z_{1}, z_{2}\left|\infty, z_{3}\right| z_{4}\right)+A_{5,2}\left(z_{1}, z_{2}\left|\infty, z_{4}\right| z_{3}\right)\right\}=\frac{1}{2} A_{4,1}\left(z_{1}, z_{2} \mid z_{3}, z_{4}\right) \\
A_{5,0}-\frac{1}{2}\left\{A_{5,1}\left(\infty, z_{1} \mid z_{2}, z_{3}, z_{4}\right)+A_{5,1}\left(\infty, z_{2} \mid z_{1}, z_{3}, z_{4}\right)+\right. \\
\left.+A_{5,1}\left(\infty, z_{3} \mid z_{1}, z_{2}, z_{4}\right)+A_{5,1}\left(\infty, z_{4} \mid z_{1}, z_{2}, z_{3}\right)\right\}=\frac{1}{2} A_{4,0}
\end{gathered}
$$

The Property 2 (3.24) for $n=5$ is equivalent to the following set of five equations

$$
\begin{gathered}
A_{5,2}\left(z_{1}, z_{2}\left|z_{3}, z_{4}\right| z_{4}+i\right)+\gamma\left(z_{34}\right) A_{5,2}\left(z_{1}, z_{2}\left|z_{3}, z_{4}+i\right| z_{4}\right)=0 \\
\gamma\left(z_{14}\right) A_{5,2}\left(z_{1}, z_{4}+i\left|z_{2}, z_{4}\right| z_{3}\right)+\gamma\left(z_{24}\right) A_{5,2}\left(z_{1}, z_{4}\left|z_{2}, z_{4}+i\right| z_{3}\right)=0
\end{gathered}
$$




$$
\begin{gathered}
\alpha\left(z_{34}\right) A_{5,2}\left(z_{1}, z_{2}\left|z_{3}, z_{4}+i\right| z_{4}\right)-2 A_{5,2}\left(z_{1}, z_{2}\left|z_{4}+i, z_{4}\right| z_{3}\right)+A_{5,1}\left(z_{1}, z_{2} \mid z_{3}, z_{4}, z_{4}+i\right)=0 \\
\alpha\left(z_{24}\right) A_{5,2}\left(z_{1}, z_{4}\left|z_{2}, z_{4}+i\right| z_{3}\right)+\alpha\left(z_{34}\right) A_{5,2}\left(z_{1}, z_{4}\left|z_{4}+i, z_{3}\right| z_{2}\right)+A_{5,1}\left(z_{1}, z_{4} \mid z_{2}, z_{3}, z_{4}+i\right)+ \\
\gamma\left(z_{14}\right) A_{5,1}\left(z_{1}, z_{4}+i \mid z_{2}, z_{3}, z_{4}\right)=0 \\
A_{5,0}+\alpha\left(z_{14}\right) A_{5,1}\left(z_{1}, z_{4}+i \mid z_{2}, z_{3}, z_{4}\right)+\alpha\left(z_{24}\right) A_{5,1}\left(z_{2}, z_{4}+i \mid z_{1}, z_{3}, z_{4}\right)+ \\
+\alpha\left(z_{34}\right) A_{5,1}\left(z_{3}, z_{4}+i \mid z_{1}, z_{2}, z_{4}\right)-2 A_{5,1}\left(z_{4}, z_{4}+i \mid z_{1}, z_{2}, z_{3}\right)=0
\end{gathered}
$$

The difference with above cases $n=2,3,4$ is that now up to the moment we do not have a solution to these equations. Therefore we have to get it. Let us briefly describe how we do this. First we take the ansatz (3.21-3.23), namely,

$$
\begin{aligned}
A_{5,0} & =\frac{1}{6} \\
A_{5,1}\left(z_{1}, z_{2} \mid z_{3}, z_{4}, z_{5}\right) & =\frac{\bar{Q}_{5,1}\left(z_{1}, z_{2} \mid z_{3}, z_{4}, z_{5}\right)}{z_{13} z_{14} z_{15} z_{23} z_{24} z_{25}} \\
A_{5,2}\left(z_{1}, z_{2}\left|z_{3}, z_{4}\right| z_{5}\right)= & \frac{\bar{Q}_{5,2}\left(z_{1}, z_{2}\left|z_{3}, z_{4}\right| z_{5}\right)}{z_{13} z_{14} z_{15} z_{23} z_{24} z_{25} z_{35} z_{45}}
\end{aligned}
$$

and demand that the polynomials $\bar{Q}_{5,1}\left(z_{1}, z_{2} \mid z_{3}, z_{4}, z_{5}\right)$ and $\bar{Q}_{5,2}\left(z_{1}, z_{2}\left|z_{3}, z_{4}\right| z_{5}\right)$ have the same symmetry properties as the functions $A_{5,1}$ and $A_{5,2}$ and the same maximum powers of the variables $z_{1}, \ldots, z_{5}$ as their denominators in the r.h.s. of (3.40) and (3.41) respectively. Namely, we write

$$
\begin{aligned}
& \bar{Q}_{5,1}\left(z_{1}, z_{2} \mid z_{3}, z_{4}, z_{5}\right)=\sum_{\substack{0 \leq i_{1}, i_{2} \leq 3, \quad 0 \leq i_{3}, i_{4}, i_{5} \leq 2 \\
i_{1}+\ldots+i_{5} \leq 6}} C_{5,1}\left(i_{1}, i_{2} \mid i_{3}, i_{4}, i_{5}\right) z_{1}^{i_{1}} z_{2}^{i_{2}} z_{3}^{i_{3}} z_{4}^{i_{4}} z_{5}^{i_{5}} \\
& \bar{Q}_{5,2}\left(z_{1}, z_{2}\left|z_{3}, z_{4}\right| z_{5}\right)=\sum_{0 \leq i_{1}, i_{2}, i_{3}, i_{4} \leq 3,} C_{5,2}\left(i_{1}, i_{2}\left|i_{3}, i_{4}\right| i_{5}\right) z_{1}^{i_{1}} z_{2}^{i_{2}} z_{3}^{i_{3}} z_{4}^{i_{4}} z_{5}^{i_{5}} \\
& 0 \leq i_{1}, i_{2}, i_{3}, i_{4} \leq 3, \quad 0 \leq i_{5} \leq 4 \\
& i_{1}+\ldots+i_{5} \leq 8
\end{aligned}
$$

with the coefficients $C_{5,1}\left(i_{1}, i_{2} \mid i_{3}, i_{4}, i_{5}\right)$ and $C_{5,2}\left(i_{1}, i_{2}\left|i_{3}, i_{4}\right| i_{5}\right)$ which have evident symmetry properties

$$
C_{5,1}\left(i_{1}, i_{2} \mid i_{3}, i_{4}, i_{5}\right)=C_{5,1}\left(i_{2}, i_{1} \mid i_{3}, i_{4}, i_{5}\right)=C_{5,1}\left(i_{1}, i_{2} \mid \sigma\left(i_{3}\right), \sigma\left(i_{4}\right), \sigma\left(i_{5}\right)\right)
$$

where $\sigma$ is any element of the permutation group of three elements $S_{3}$ while

$$
C_{5,2}\left(i_{1}, i_{2}\left|i_{3}, i_{4}\right| i_{5}\right)=C_{5,2}\left(i_{2}, i_{1}\left|i_{3}, i_{4}\right| i_{5}\right)=C_{5,2}\left(i_{1}, i_{2}\left|i_{4}, i_{3}\right| i_{5}\right)=C_{5,2}\left(i_{3}, i_{4}\left|i_{1}, i_{2}\right| i_{5}\right)
$$

In accordance with eq. (3.19) we also demand the translational invariance of these polynomials

$$
\begin{aligned}
& \bar{Q}_{5,1}\left(z_{1}+a, z_{2}+a \mid z_{3}+a, z_{4}+a, z_{5}+a\right)=\bar{Q}_{5,1}\left(z_{1}, z_{2} \mid z_{3}, z_{4}, z_{5}\right) \\
& \bar{Q}_{5,2}\left(z_{1}+a, z_{2}+a\left|z_{3}+a, z_{4}+a\right| z_{5}+a\right)=\bar{Q}_{5,2}\left(z_{1}, z_{2}\left|z_{3}, z_{4}\right| z_{5}\right)
\end{aligned}
$$


These conditions allow us to fix some of the coefficients $C_{5,1}$ and $C_{5,2}$ in (3.42-3.43).

The rest of these coefficients may be fixed by satisfying the equations (3.38). One should say that in spite of the fact that it turns out to be an over determined system it has a solution and this solution is unique. Also it is interesting to note that after the equations (3.38) are satisfied all the residual equations (3.37) are satisfied automatically (!).

Let us show the result

$$
\begin{gathered}
\bar{Q}_{5,2}\left(z_{1}, z_{2}\left|z_{3}, z_{4}\right| z_{5}\right)= \\
\frac{1}{2}\left(z_{15} z_{25}-1\right)\left(z_{35} z_{45}-1\right) \bar{Q}_{4,2}\left(z_{1}, z_{2} \mid z_{3}, z_{4}\right)+\frac{1}{360}\left(z_{12}^{2}+4\right)\left(z_{34}^{2}+4\right)\left(5-z_{13} z_{24}-z_{14} z_{23}\right) \\
\bar{Q}_{5,1}\left(z_{1}, z_{2} \mid z_{3}, z_{4}, z_{5}\right)= \\
\frac{1}{2}\left(z_{15} z_{25}-1\right) \bar{Q}_{4,2}\left(z_{1}, z_{2} \mid z_{3}, z_{4}\right)+\frac{1}{2}\left(z_{14} z_{24}-1\right) \bar{Q}_{4,2}\left(z_{1}, z_{2} \mid z_{3}, z_{5}\right)+\frac{1}{2}\left(z_{13} z_{23}-1\right) \bar{Q}_{4,2}\left(z_{1}, z_{2} \mid z_{4}, z_{5}\right) \\
-\frac{\left(z_{12}^{2}+4\right)}{360}\left\{22+\left(z_{34}^{2}-2\right)\left(z_{15} z_{25}+\frac{1}{2}\right)+\left(z_{35}^{2}-2\right)\left(z_{14} z_{24}+\frac{1}{2}\right)+\left(z_{45}^{2}-2\right)\left(z_{13} z_{23}+\frac{1}{2}\right)\right\}
\end{gathered}
$$

Now using eqs. (3.39-3.41) and substituting these formulae into the ansatz (3.20) we get the answer for $P_{5}$ in the inhomogeneous case.

The case $n=6$

We proceed in the same line as for the case $n=5$. The Property 1 i.e. eq.(3.25) or equivalently the set of the recurrent relations (3.28) at $k=3$ gives us three equations

$$
\begin{gathered}
A_{6,2}\left(z_{1}, z_{2}\left|z_{3}, z_{4}\right| z_{5}, \infty\right)-\frac{1}{2} A_{6,3}\left(z_{1}, z_{2}\left|z_{3}, z_{4}\right| z_{5}, \infty\right)=\frac{1}{2} A_{5,2}\left(z_{1}, z_{2}\left|z_{3}, z_{4}\right| z_{5}\right) \\
A_{6,1}\left(z_{1}, z_{2} \mid z_{3}, z_{4}, z_{5}, \infty\right)-\frac{1}{2}\left\{A_{6,2}\left(z_{1}, z_{2}\left|\infty, z_{3}\right| z_{4}, z_{5}\right)+A_{6,2}\left(z_{1}, z_{2}\left|\infty, z_{4}\right| z_{3}, z_{5}\right)+\right. \\
\left.+A_{6,2}\left(z_{1}, z_{2}\left|\infty, z_{5}\right| z_{3}, z_{4}\right)\right\}=\frac{1}{2} A_{5,1}\left(z_{1}, z_{2} \mid z_{3}, z_{4}, z_{5}\right) \\
A_{6,0}-\frac{1}{2}\left\{A_{6,1}\left(\infty, z_{1} \mid z_{2}, z_{3}, z_{4}, z_{5}\right)+A_{6,1}\left(\infty, z_{2} \mid z_{1}, z_{3}, z_{4}, z_{5}\right)+A_{6,1}\left(\infty, z_{3} \mid z_{1}, z_{2}, z_{4}, z_{5}\right)+\right. \\
\left.+A_{6,1}\left(\infty, z_{4} \mid z_{1}, z_{2}, z_{3}, z_{5}\right)+A_{6,1}\left(\infty, z_{5} \mid z_{1}, z_{2}, z_{3}, z_{4}\right)\right\}=\frac{1}{2} A_{50}
\end{gathered}
$$

The Property $2(3.24)$ for $n=6$ produces seven equations

$$
\begin{gathered}
A_{6,2}\left(z_{1}, z_{2}\left|z_{3}, z_{4}\right| z_{5}, z_{5}+i\right)-2 A_{6,3}\left(z_{1}, z_{2}\left|z_{3}, z_{4}\right| z_{5}, z_{5}+i\right)=0 \\
\gamma\left(z_{45}\right) A_{6,3}\left(z_{1}, z_{2}\left|z_{3}, z_{5}\right| z_{4}, z_{5}+i\right)+\gamma\left(z_{35}\right) A_{6,3}\left(z_{1}, z_{2}\left|z_{3}, z_{5}+i\right| z_{4}, z_{5}\right)=0 \\
A_{6,2}\left(z_{1}, z_{2}\left|z_{3}, z_{5}\right| z_{4}, z_{5}+i\right)+\gamma\left(z_{35}\right) A_{6,2}\left(z_{1}, z_{2}\left|z_{3}, z_{5}+i\right| z_{4}, z_{5}\right)+ \\
+\alpha\left(z_{45}\right) A_{6,3}\left(z_{1}, z_{2}\left|z_{3}, z_{5}\right| z_{4}, z_{5}+i\right)=0 \\
\alpha\left(z_{35}\right) A_{6,2}\left(z_{1}, z_{2}\left|z_{3}, z_{5}+i\right| z_{4}, z_{5}\right)+\alpha\left(z_{45}\right) A_{6,2}\left(z_{1}, z_{2}\left|z_{4}, z_{5}+i\right| z_{3}, z_{5}\right)-
\end{gathered}
$$




$$
\begin{gathered}
-2 A_{6,2}\left(z_{1}, z_{2}\left|z_{5}, z_{5}+i\right| z_{3}, z_{4}\right)+A_{6,1}\left(z_{1}, z_{2} \mid z_{3}, z_{4}, z_{5}, z_{5}+i\right)=0 \\
\gamma\left(z_{25}\right) A_{6,2}\left(z_{1}, z_{5}\left|z_{2}, z_{5}+i\right| z_{3}, z_{4}\right)+\gamma\left(z_{15}\right) A_{6,2}\left(z_{1}, z_{5}+i\left|z_{2}, z_{5}\right| z_{3}, z_{4}\right)=0 \\
A_{6,1}\left(z_{1}, z_{5} \mid z_{2}, z_{3}, z_{4}, z_{5}+i\right)+\gamma\left(z_{15}\right) A_{6,1}\left(z_{1}, z_{5}+i \mid z_{2}, z_{3}, z_{4}, z_{5}\right)+ \\
\alpha\left(z_{25}\right) A_{6,2}\left(z_{1}, z_{5}\left|z_{2}, z_{5}+i\right| z_{3}, z_{4}\right)+\alpha\left(z_{35}\right) A_{6,2}\left(z_{1}, z_{5}\left|z_{3}, z_{5}+i\right| z_{2}, z_{4}\right)+ \\
+\alpha\left(z_{45}\right) A_{6,2}\left(z_{1}, z_{5}\left|z_{4}, z_{5}+i\right| z_{2}, z_{3}\right)=0 \\
A_{6,0}-2 A_{6,1}\left(z_{5}, z_{5}+i \mid z_{1}, z_{2}, z_{3}, z_{4}\right)+\alpha\left(z_{15}\right) A_{6,1}\left(z_{1}, z_{5}+i \mid z_{2}, z_{3}, z_{4}, z_{5}\right)+ \\
\alpha\left(z_{25}\right) A_{6,1}\left(z_{2}, z_{5}+i \mid z_{1}, z_{3}, z_{4}, z_{5}\right)+\alpha\left(z_{35}\right) A_{6,1}\left(z_{3}, z_{5}+i \mid z_{1}, z_{2}, z_{4}, z_{5}\right)+ \\
+\alpha\left(z_{45}\right) A_{6,1}\left(z_{4}, z_{5}+i \mid z_{1}, z_{2}, z_{3}, z_{4}\right)=0
\end{gathered}
$$

In the same line as we did it above for the case $n=5$ we can solve the equations (3.50) and (3.51) in framework of the ansatz (3.21-3.23)

$$
\begin{gathered}
A_{6,0}=\frac{1}{7} \\
A_{6,1}\left(z_{1}, z_{2} \mid z_{3}, z_{4}, z_{5}, z_{6}\right)=\frac{\bar{Q}_{6,1}\left(z_{1}, z_{2} \mid z_{3}, z_{4}, z_{5}, z_{6}\right)}{z_{13} z_{14} z_{15} z_{16} z_{23} z_{24} z_{25} z_{26}} \\
A_{6,2}\left(z_{1}, z_{2}\left|z_{3}, z_{4}\right| z_{5}, z_{6}\right)=\frac{\bar{Q}_{6,2}\left(z_{1}, z_{2}\left|z_{3}, z_{4}\right| z_{5}, z_{6}\right)}{z_{13} z_{14} z_{15} z_{16} z_{23} z_{24} z_{25} z_{26} z_{35} z_{36} z_{45} z_{46}} \\
A_{6,3}\left(z_{1}, z_{2}\left|z_{3}, z_{4}\right| z_{5}, z_{6}\right)=\frac{\bar{Q}_{6,3}\left(z_{1}, z_{2}\left|z_{3}, z_{4}\right| z_{5}, z_{6}\right)}{z_{13} z_{14} z_{15} z_{16} z_{23} z_{24} z_{25} z_{26} z_{35} z_{36} z_{45} z_{46}}
\end{gathered}
$$

As above we demand that the polynomials $\bar{Q}_{6,1}\left(z_{1}, z_{2} \mid z_{3}, z_{4}, z_{5}, z_{6}\right), \bar{Q}_{6,2}\left(z_{1}, z_{2}\left|z_{3}, z_{4}\right| z_{5}, z_{6}\right)$ and $\bar{Q}_{6,3}\left(z_{1}, z_{2}\left|z_{3}, z_{4}\right| z_{5}, z_{6}\right)$ have the same symmetry properties as the functions $A_{6,1}, A_{6,2}, A_{6,3}$ respectively. Analogous to eqs. (3.42-3.43) we write

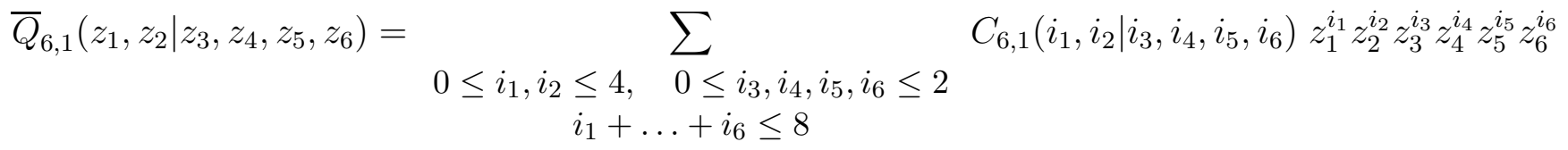

$$
\begin{aligned}
& \bar{Q}_{6,2}\left(z_{1}, z_{2}\left|z_{3}, z_{4}\right| z_{5}, z_{6}\right)=\sum_{\substack{0 \leq i_{1}, i_{2}, \ldots, i_{6} \leq 4 \\
i_{1}+\ldots+i_{6} \leq 12}} C_{6,2}\left(i_{1}, i_{2}\left|i_{3}, i_{4}\right| i_{5}, i_{6}\right) z_{1}^{i_{1}} z_{2}^{i_{2}} z_{3}^{i_{3}} z_{4}^{i_{4}} z_{5}^{i_{5}} z_{6}^{i_{6}} \\
& \bar{Q}_{6,3}\left(z_{1}, z_{2}\left|z_{3}, z_{4}\right| z_{5}, z_{6}\right)=\quad \sum \quad C_{6,3}\left(i_{1}, i_{2}\left|i_{3}, i_{4}\right| i_{5}, i_{6}\right) z_{1}^{i_{1}} z_{2}^{i_{2}} z_{3}^{i_{3}} z_{4}^{i_{4}} z_{5}^{i_{5}} z_{6}^{i_{6}} \\
& 0 \leq i_{1}, i_{2}, \ldots, i_{6} \leq 4 \\
& i_{1}+\ldots+i_{6} \leq 12
\end{aligned}
$$

with the coefficients $C_{6,1}\left(i_{1}, i_{2} \mid i_{3}, i_{4}, i_{5}, i_{6}\right), C_{6,2}\left(i_{1}, i_{2}\left|i_{3}, i_{4}\right| i_{5}, i_{6}\right)$ and $C_{6,3}\left(i_{1}, i_{2}\left|i_{3}, i_{4}\right| i_{5}, i_{6}\right)$ which satisfy the symmetry conditions

$$
C_{6,1}\left(i_{1}, i_{2} \mid i_{3}, i_{4}, i_{5}, i_{6}\right)=C_{6,1}\left(i_{2}, i_{1} \mid i_{3}, i_{4}, i_{5}, i_{6}\right)=C_{6,1}\left(i_{1}, i_{2} \mid \sigma\left(i_{3}\right), \sigma\left(i_{4}\right), \sigma\left(i_{5}\right), \sigma\left(i_{6}\right)\right)
$$


where $\sigma$ is any element of the permutation group of four elements $S_{4}$

$$
\begin{gathered}
C_{6,2}\left(i_{1}, i_{2}\left|i_{3}, i_{4}\right| i_{5}, i_{6}\right)=C_{6,2}\left(i_{2}, i_{1}\left|i_{3}, i_{4}\right| i_{5}, i_{6}\right)=C_{6,2}\left(i_{1}, i_{2}\left|i_{4}, i_{3}\right| i_{5}, i_{6}\right)=C_{6,2}\left(i_{1}, i_{2}\left|i_{3}, i_{4}\right| i_{6}, i_{5}\right)= \\
=C_{6,2}\left(i_{3}, i_{4}\left|i_{1}, i_{2}\right| i_{5}, i_{6}\right) \\
C_{6,3}\left(i_{1}, i_{2}\left|i_{3}, i_{4}\right| i_{5}, i_{6}\right)= \\
=C_{6,3}\left(i_{2}, i_{1}\left|i_{3}, i_{4}\right| i_{5}, i_{6}\right)=C_{6,3}\left(i_{1}, i_{2}\left|i_{4}, i_{3}\right| i_{5}, i_{6}\right)=C_{6,3}\left(i_{1}, i_{2}\left|i_{3}, i_{4}\right| i_{6}, i_{5}\right)= \\
=C_{6,3}\left(i_{3}, i_{4}\left|i_{1}, i_{2}\right| i_{5}, i_{6}\right)=C_{6,3}\left(i_{1}, i_{2}\left|i_{5}, i_{6}\right| i_{3}, i_{4}\right)
\end{gathered}
$$

As in the previous case the translational invariance of these polynomials

$$
\begin{aligned}
& \bar{Q}_{6,1}\left(z_{1}+a, z_{2}+a \mid z_{3}+a, z_{4}+a, z_{5}+a, z_{6}+a\right)=\bar{Q}_{6,1}\left(z_{1}, z_{2} \mid z_{3}, z_{4}, z_{5}, z_{6}\right) \\
& \bar{Q}_{6,2}\left(z_{1}+a, z_{2}+a\left|z_{3}+a, z_{4}+a\right| z_{5}+a, z_{6}+a\right)=\bar{Q}_{6,2}\left(z_{1}, z_{2}\left|z_{3}, z_{4}\right| z_{5}, z_{6}\right) \\
& \bar{Q}_{6,3}\left(z_{1}+a, z_{2}+a\left|z_{3}+a, z_{4}+a\right| z_{5}+a, z_{6}+a\right)=\bar{Q}_{6,3}\left(z_{1}, z_{2}\left|z_{3}, z_{4}\right| z_{5}, z_{6}\right)
\end{aligned}
$$

allow us to fix a lot of the coefficients $C_{6,1}, C_{6,2}$ and $C_{6,3}$.

Then with the help of computer we solve the over determined system of the equations (3.51). It is left to check that all other equations (3.50) are satisfied automatically as in the case $n=5$.

The result looks as follows

$$
\begin{gathered}
\bar{Q}_{6,3}\left(z_{1}, z_{2}\left|z_{3}, z_{4}\right| z_{5}, z_{6}\right)= \\
6^{3} \bar{Q}_{4,2}\left(z_{1}, z_{2} \mid z_{3}, z_{4}\right) \bar{Q}_{4,2}\left(z_{1}, z_{2} \mid z_{5}, z_{6}\right) \bar{Q}_{4,2}\left(z_{3}, z_{4} \mid z_{5}, z_{6}\right)+\left(z_{12}^{2}+4\right)\left(z_{34}^{2}+4\right)\left(z_{56}^{2}+4\right) \Lambda_{6,3}\left(z_{1}, z_{2}\left|z_{3}, z_{4}\right| z_{5}, z_{6}\right) \\
\bar{Q}_{6,2}\left(z_{1}, z_{2}\left|z_{3}, z_{4}\right| z_{5}, z_{6}\right)= \\
\frac{6^{3}}{2} \bar{Q}_{4,2}\left(z_{1}, z_{2} \mid z_{3}, z_{4}\right) \bar{Q}_{4,1}\left(z_{1}, z_{2} \mid z_{5}, z_{6}\right) \bar{Q}_{4,1}\left(z_{3}, z_{4} \mid z_{5}, z_{6}\right)+\left(z_{12}^{2}+4\right)\left(z_{34}^{2}+4\right) \Lambda_{6,2}\left(z_{1}, z_{2}\left|z_{3}, z_{4}\right| z_{5}, z_{6}\right) \\
\bar{Q}_{6,1}\left(z_{1}, z_{2} \mid z_{3}, z_{4}, z_{5}, z_{6}\right)= \\
\frac{1}{30}\left(z_{13} z_{23}-1\right)\left(z_{14} z_{24}-1\right)\left(z_{15} z_{25}-1\right)\left(z_{16} z_{26}-1\right)+\left(z_{12}^{2}+4\right) \Lambda_{6,1}\left(z_{1}, z_{2} \mid z_{3}, z_{4}, z_{5}, z_{6}\right)
\end{gathered}
$$

where

$$
\begin{gathered}
\Lambda_{6,3}\left(z_{1}, z_{2}\left|z_{3}, z_{4}\right| z_{5}, z_{6}\right)=\frac{1}{5400}\left(5-z_{13} z_{24}-z_{14} z_{23}\right)\left(5-z_{15} z_{26}-z_{16} z_{25}\right)\left(5-z_{35} z_{46}-z_{36} z_{45}\right) \\
-\frac{1}{189000}\left(z_{12}^{2}-26\right)\left(z_{34}^{2}-26\right)\left(z_{56}^{2}-26\right)+\frac{1}{200}\left(z_{12}^{2}+z_{34}^{2}+z_{56}^{2}-16\right) \\
\Lambda_{6,2}\left(z_{1}, z_{2}\left|z_{3}, z_{4}\right| z_{5}, z_{6}\right)=\left\{\frac{1}{2} \Lambda_{6,3}\left(z_{1}, z_{2}\left|z_{3}, z_{4}\right| z_{5}, z_{6}\right)+\frac{1}{100} \bar{Q}_{42}\left(z_{1}, z_{2} \mid z_{3}, z_{4}\right)\left(z_{56}^{2}+1\right)\right\}\left(z_{56}^{2}+13\right)+ \\
\left\{\frac{1}{720}\left(5-z_{13} z_{24}-z_{14} z_{23}\right)\left(z_{56}^{2}-3+z_{15} z_{26}+z_{16} z_{25}+z_{35} z_{46}+z_{36} z_{45}\right)-\right. \\
\left.-\frac{1}{2520}\left(z_{12}^{2}+\frac{11}{2}\right)\left(z_{34}^{2}+\frac{11}{2}\right)-\frac{1}{160}\right\}\left(z_{56}^{2}+1\right)
\end{gathered}
$$




$$
\begin{gathered}
\Lambda_{6,1}\left(z_{1}, z_{2} \mid z_{3}, z_{4}, z_{5}, z_{6}\right)=\frac{1}{600}\left(z_{13} z_{14} z_{23} z_{24} z_{56}^{2}+z_{13} z_{15} z_{23} z_{25} z_{46}^{2}+\ldots+z_{15} z_{16} z_{25} z_{26} z_{34}^{2}\right)+ \\
+\frac{11}{900}\left(z_{13} z_{14} z_{23} z_{24}+z_{13} z_{15} z_{23} z_{25}+\ldots+z_{15} z_{16} z_{25} z_{26}\right)- \\
-\frac{1}{225}\left\{\left(z_{13} z_{24}+z_{14} z_{23}\right) z_{56}^{2}+\left(z_{13} z_{25}+z_{15} z_{23}\right) z_{46}^{2}+\ldots+\left(z_{15} z_{26}+z_{16} z_{25}\right) z_{34}^{2}\right\}- \\
-\frac{7}{300}\left\{\left(z_{13} z_{24}+z_{14} z_{23}\right)+\left(z_{13} z_{25}+z_{15} z_{23}\right)+\ldots+\left(z_{15} z_{26}+z_{16} z_{25}\right)\right\}+\frac{1}{8400} z_{12}^{2}\left(z_{34}^{2} z_{56}^{2}+z_{35}^{2} z_{46}^{2}+z_{36}^{2} z_{45}^{2}\right)- \\
-\frac{19}{2520}\left(z_{34}^{2} z_{56}^{2}+z_{35}^{2} z_{46}^{2}+z_{36}^{2} z_{45}^{2}\right)+\frac{1}{1575} z_{12}^{2}\left(z_{34}^{2}+z_{56}^{2}+z_{35}^{2}+z_{46}^{2}+z_{36}^{2}+z_{45}^{2}\right)- \\
-\frac{1}{168}\left(z_{34}^{2}+z_{56}^{2}+z_{35}^{2}+z_{46}^{2}+z_{36}^{2}+z_{45}^{2}\right)+\frac{13}{840} z_{12}^{2}+\frac{29}{70}
\end{gathered}
$$

By means of this solution, eqs. (3.52-3.55) and the ansatz (3.20) for $n=6$ we come (for the very first time (!)) to the result for $P_{6}$ in the inhomogeneous case.

Let us make a remark about some additional amusing factorization property of the polynomials $\bar{Q}_{n l}$ whose meaning is still to realize, namely

$$
\begin{gathered}
\bar{Q}_{3,1}\left(z_{1}, z_{1}+2 i \mid z_{3}\right)=\frac{1}{12}\left(z_{13}+i\right)^{2} \\
\bar{Q}_{4,2}\left(z_{1}, z_{1}+2 i \mid z_{3}, z_{4}\right)=\frac{1}{36}\left(z_{13}+i\right)^{2}\left(z_{14}+i\right)^{2} \\
\bar{Q}_{4,1}\left(z_{1}, z_{1}+2 i \mid z_{3}, z_{4}\right)=\frac{1}{18}\left(z_{13}+i\right)^{2}\left(z_{14}+i\right)^{2} \\
\bar{Q}_{5,2}\left(z_{1}, z_{1}+2 i\left|z_{3}, z_{4}\right| z_{5}\right)=\frac{1}{6}\left(z_{13}+i\right)^{2}\left(z_{14}+i\right)^{2}\left(z_{15}+i\right)^{2} \bar{Q}_{31}\left(z_{3}, z_{4} \mid z_{5}\right) \\
\bar{Q}_{5,1}\left(z_{1}, z_{1}+2 i\left|z_{3}, z_{4}\right| z_{5}\right)=\frac{1}{24}\left(z_{13}+i\right)^{2}\left(z_{14}+i\right)^{2}\left(z_{15}+i\right)^{2} \\
\bar{Q}_{6,3}\left(z_{1}, z_{1}+2 i\left|z_{3}, z_{4}\right| z_{5}, z_{6}\right)=\frac{1}{6}\left(z_{13}+i\right)^{2}\left(z_{14}+i\right)^{2}\left(z_{15}+i\right)^{2}\left(z_{16}+i\right)^{2} \bar{Q}_{4,2}\left(z_{3}, z_{4} \mid z_{5}, z_{6}\right) \\
\bar{Q}_{6,2}\left(z_{1}, z_{1}+2 i\left|z_{3}, z_{4}\right| z_{5}, z_{6}\right)=\frac{1}{6}\left(z_{13}+i\right)^{2}\left(z_{14}+i\right)^{2}\left(z_{15}+i\right)^{2}\left(z_{16}+i\right)^{2} \bar{Q}_{4,1}\left(z_{3}, z_{4} \mid z_{5}, z_{6}\right) \\
\bar{Q}_{6,1}\left(z_{1}, z_{1}+2 i\left|z_{3}, z_{4}\right| z_{5}, z_{6}\right)=\frac{1}{30}\left(z_{13}+i\right)^{2}\left(z_{14}+i\right)^{2}\left(z_{15}+i\right)^{2}\left(z_{16}+i\right)^{2}
\end{gathered}
$$

Looking at these formulae we can assume that

$$
\frac{\bar{Q}_{n, l}\left(z_{1}, z_{1}+2 i\left|z_{3}, z_{4}\right| \ldots\left|z_{2 l-1} z_{2 l}\right| z_{2 l+1} \ldots z_{n}\right)}{\bar{Q}_{n-2, l-1}\left(z_{3}, z_{4}|\ldots| z_{2 l-1} z_{2 l} \mid z_{2 l+1} \ldots z_{n}\right)}=\frac{1}{6} \prod_{j=3}^{n}\left(z_{1 j}+i\right)^{2}
$$




\section{The structure of $P(n)$ in homogeneous case}

As we have discussed in [10 the formulae in the homogeneous case become more regular in terms of the alternating zeta values

$$
\zeta_{a}(s)=\sum_{n>0} \frac{(-1)^{n-1}}{n^{s}}=-\mathrm{Li}_{s}(-1)
$$

where $\operatorname{Li}_{s}(x)$ is the polylogarithm. The alternating zeta series is related to the Riemann zeta function as follows

$$
\zeta(s)=\frac{1}{1-2^{1-s}} \zeta_{a}(s)
$$

Unlike Riemann zeta function the alternating zeta series is regular at $s=1$, it is $\zeta_{a}(1)=\log 2$. Let us mention that the answers for $P(1), \ldots, P(4)$ can be obtained from the formulae (3.2-3.5) by taking the homogeneous limit i.e. $z_{j} \rightarrow 0$ and using the expansion of the function $G$ (3.13).

The same can be also done for $n=5$ and $n=6$ by means of the formula (320), formulae (3.21), (3.22), (3.23) and the answers for the polynomials (3.48-3.49) and (3.65-3.67) for the cases $n=5$ and $n=6$ respectively.

For $n \leq 5$ we reproduce the known results, see [10] formula (1.16) in there.

In the case $n=6$ we discover a new result

$$
\begin{gathered}
P(6)=\frac{1}{7}\left\{1-35 \zeta_{a}(1)+322 \zeta_{a}(3)-\frac{9244}{5} \zeta_{a}(5)+\frac{22694}{5} \zeta_{a}(7)-2982 \zeta_{a}(9)\right. \\
-\frac{3920}{3} \zeta_{a}(1) \cdot \zeta_{a}(3)+\frac{369908}{15} \zeta_{a}(1) \cdot \zeta_{a}(5)-\frac{28784}{5} \zeta_{a}(3)^{2}-\frac{263816}{3} \zeta_{a}(1) \cdot \zeta_{a}(7) \\
+\frac{3458}{15} \zeta_{a}(3) \cdot \zeta_{a}(5)+\frac{323344}{5} \zeta_{a}(1) \cdot \zeta_{a}(9)+\frac{933702}{5} \zeta_{a}(3) \cdot \zeta_{a}(7)-\frac{751592}{9} \zeta_{a}(5)^{2} \\
-\frac{2627842}{15} \zeta_{a}(3) \cdot \zeta_{a}(9)+\frac{235963}{9} \zeta_{a}(5) \cdot \zeta_{a}(7)+\frac{368564}{3} \zeta_{a}(5) \cdot \zeta_{a}(9)-\frac{644987}{9} \zeta_{a}(7)^{2} \\
+\frac{538496}{45} \zeta_{a}(1) \cdot \zeta_{a}(3) \cdot \zeta_{a}(5)-\frac{269248}{135} \zeta_{a}(3)^{3}-\frac{1143268}{9} \zeta_{a}(1) \cdot \zeta_{a}(3) \cdot \zeta_{a}(7)+\frac{653296}{9} \zeta_{a}(1) \cdot \zeta_{a}(5)^{2} \\
-\frac{163324}{45} \zeta_{a}(3)^{2} \cdot \zeta_{a}(5)+\frac{1737148}{15} \zeta_{a}(1) \cdot \zeta_{a}(3) \cdot \zeta_{a}(9)-\frac{1737148}{45} \zeta_{a}(3)^{2} \cdot \zeta_{a}(7)+\frac{124082}{9} \zeta_{a}(3) \cdot \zeta_{a}(5)^{2} \\
-\frac{528164}{3} \zeta_{a}(1) \cdot \zeta_{a}(5) \cdot \zeta_{a}(9)+\frac{924287}{9} \zeta_{a}(1) \cdot \zeta_{a}(7)^{2}+\frac{264082}{5} \zeta_{a}(3)^{2} \cdot \zeta_{a}(9) \\
\left.-\frac{264082}{9} \zeta_{a}(3) \cdot \zeta_{a}(5) \cdot \zeta_{a}(7)+\frac{188630}{27} \zeta_{a}(5)^{3}\right\}
\end{gathered}
$$

This support our hypothesis that all $P(n)$ can be expression in terms of values of Riemann zeta functions at odd arguments, $\log 2$ and rational coefficients.

From this expression we can get numerical value

$$
P(6)=7.068127533 \cdot 10^{-9}
$$

In [10] a numerical method was used for evaluation of $P(6)$. It is called Density Matrix Renormalization Group (DMRG). The results can be found in Table 1 in 10 . In particular $P(6)=7.05 \cdot 10^{-9}$ with an uncertainty in the second digit after the decimal point. It is in a good agreement with our analytic result (4.3), (4.4). 
Looking at the formulae (1.16) of [10] and the above expression (4.3) we can make a general conjecture for the dependence of $P(n)$ on the alternating zeta series

$$
P(n)=\frac{1}{(n+1)} \sum_{\vec{r} \in U} B_{r_{0}, r_{1}, \ldots, r_{n-2}}^{(n)} \prod_{j=0}^{n-2}\left[\zeta_{a}(2 j+1)\right]^{r_{j}}
$$

where all coefficients $B_{r_{0}, r_{1}, \ldots, r_{n-2}}^{(n)}$ are rational and the sum is over non-negative integers $r_{0}, \ldots, r_{n-2}$ which belong to the region $U$ determined by the following two conditions

$$
\begin{gathered}
\sum_{j=0}^{n-2} r_{j} \leq[n / 2] \\
\sum_{j=0}^{n-2} r_{j}(2 j+1) \leq \frac{n(n-1)}{2}
\end{gathered}
$$

Let us show how the non-zero coefficients $B_{r_{0}, r_{1}, \ldots, r_{n-2}}^{(n)}$ look like for the cases when we know the manifest analytic answer, namely, when $n=1,2, \ldots 6$

$$
\begin{aligned}
& B^{(1)}=1 \\
& B_{0}^{(2)}=1 \quad B_{1}^{(2)}=-1 \\
& B_{0,0}^{(3)}=1 \quad B_{1,0}^{(3)}=-4 \quad B_{0,1}^{(3)}=2 \\
& B_{0,0,0}^{(4)}=1 \quad B_{1,0,0}^{(4)}=-10 \quad B_{0,1,0}^{(4)}=\frac{173}{9} \quad B_{0,0,1}^{(4)}=-\frac{110}{9} \\
& B_{1,1,0}^{(4)}=-\frac{110}{9} \quad B_{1,0,1}^{(4)}=\frac{170}{9} \quad B_{0,2,0}^{(4)}=-\frac{17}{3} \\
& B_{0,0,0,0}^{(5)}=1 \quad B_{1,0,0,0}^{(5)}=-20 \quad B_{0,1,0,0}^{(5)}=\frac{281}{3} \quad B_{0,0,1,0}^{(5)}=-\frac{1355}{6} \quad B_{0,0,0,1}^{(5)}=\frac{889}{6} \\
& B_{1,1,0,0}^{(5)}=-180 \quad B_{1,0,1,0}^{(5)}=\frac{3920}{3} \quad B_{1,0,0,1}^{(5)}=-\frac{3290}{3} \quad B_{0,1,1,0}^{(5)}=-\frac{170}{3} \quad B_{0,1,0,1}^{(5)}=679 \\
& B_{0,2,0,0}^{(5)}=-326 \quad B_{0,0,2,0}^{(5)}=-\frac{970}{3} \\
& B_{0,0,0,0,0}^{(6)}=1 \\
& B_{1,0,0,0,0}^{(6)}=-35 \quad B_{0,1,0,0,0}^{(6)}=322 \quad B_{0,0,1,0,0}^{(6)}=-\frac{9244}{5} \quad B_{0,0,0,1,0}^{(6)}=\frac{22694}{5} \quad B_{0,0,0,0,1}^{(6)}=-2982 \\
& B_{1,1,0,0,0}^{(6)}=-\frac{3920}{3} \quad B_{1,0,1,0,0}^{(6)}=\frac{369908}{15} \quad B_{1,0,0,1,0}^{(6)}=-\frac{263816}{3} \quad B_{1,0,0,0,1}^{(6)}=\frac{323344}{5} \\
& B_{0,1,1,0,0}^{(6)}=\frac{3458}{15} \quad B_{0,1,0,1,0}^{(6)}=\frac{933702}{5} \quad B_{0,1,0,0,1}^{(6)}=-\frac{2627842}{15} \quad B_{0,0,1,1,0}^{(6)}=\frac{235963}{9} \\
& B_{0,0,1,0,1}^{(6)}=\frac{368564}{3} \quad B_{0,2,0,0,0}^{(6)}=-\frac{28784}{5} \quad B_{0,0,2,0,0}^{(6)}=-\frac{751592}{9} \quad B_{0,0,0,2,0}^{(6)}=-\frac{644987}{9} \\
& B_{1,1,1,0,0}^{(6)}=\frac{538496}{45} \quad B_{1,1,0,1,0}^{(6)}=-\frac{1143268}{9} \quad B_{1,1,0,0,1}^{(6)}=\frac{1737148}{15} \quad B_{1,0,1,0,1}^{(6)}=-\frac{528164}{3}
\end{aligned}
$$




$$
\begin{gathered}
B_{0,1,1,1,0}^{(6)}=-\frac{264082}{9} \quad B_{1,0,2,0,0}^{(6)}=\frac{653296}{9} \quad B_{1,0,0,2,0}^{(6)}=\frac{924287}{9} \quad B_{0,1,2,0,0}^{(6)}=\frac{124082}{9} \\
B_{0,2,1,0,0}^{(6)}=-\frac{163324}{45} \quad B_{0,2,0,1,0}^{(6)}=-\frac{1737148}{45} \quad B_{0,2,0,0,1}^{(6)}=\frac{264082}{5} \\
B_{0,3,0,0,0}^{(6)}=-\frac{269248}{135} \quad B_{0,0,3,0,0}^{(6)}=\frac{188630}{27}
\end{gathered}
$$

For us it was a bit surprising that two of coefficients $B^{(6)}$ appeared to be zero, namely,

$$
B_{1,0,1,1,0}^{(6)}=0 \quad B_{1,2,0,0,0}^{(6)}=0
$$

It means that the structures $\zeta_{a}(1) \cdot \zeta_{a}(5) \cdot \zeta_{a}(7)$ and $\zeta_{a}(1) \cdot \zeta_{a}(3)^{2}$ do not appear in the final answer for $P(6)$. Meanwhile the term $\zeta_{a}(3)^{3}$ survived with the non-zero coefficient $B_{0,3,0,0,0}^{(6)}$ which we expected to be zero.

The most evident conjecture that we can make looking at the formulae (4.7) is as follows

$$
\begin{gathered}
B_{0,0, \ldots, 0}^{(n)}=1, \\
B_{1,0, \ldots, 0}^{(n)}=-\left(\begin{array}{c}
n+1 \\
3
\end{array}\right)
\end{gathered}
$$

where $\left(\begin{array}{c}n \\ m\end{array}\right)$ is the binomial coefficient.

The next our conjecture is less trivial. As appeared the coefficients $B$ might satisfy some equations. One of them has the following form

$$
\sum_{r_{0}+r_{1}+\ldots+r_{n-2}=p} B_{r_{0}, r_{1}, \ldots, r_{n-2}}^{(n)}=(-1)^{p}\left(\begin{array}{c}
n-p \\
p
\end{array}\right)
$$

where $p$ is some fixed positive integer. The expression (4.11) can be easily verified for the first coefficients given by the formulae (4.7). For example, when $p=1$ we get the following equation

$$
B_{1,0, \ldots, 0}^{(n)}+B_{0,1, \ldots, 0}^{(n)}+\ldots+B_{0,0, \ldots, 1}^{(n)}=-n+1
$$

where we have already made the conjecture (4.10) for the first term. We believe that there should be more equations like (4.11) which probably provide the rigorous expression for the coefficients $B$.

We have pointed out in our previous work [10] that since $\zeta_{a}(1), \zeta_{a}(3), \zeta_{a}(5), \ldots$ are very likely different irrational (or even transcendental) numbers 0 国 $P(n)$ seem to be different irrational (or transcendental) numbers as well. This means that $P(n)$ does not satisfy polynomial recursion relation with respect to the distance.

In order to clarify the structure of the formula (4.5) let us formally replace all $\zeta_{a}(2 j+1)$ by one complex variable $x$. This will define a new function $P(n, x)$.

Then we can calculate $P(n, x)$ using our conjectures (4.5), (4.11) and some properties of the binomial coefficients. Namely,

$$
P(n, x)=\sum_{p=0}^{[n / 2]}(-x)^{p}\left(\begin{array}{c}
n-p \\
p
\end{array}\right)=\frac{A_{+}^{n+1}-A_{-}^{n+1}}{(n+1) \sqrt{1-4 x}}
$$

\footnotetext{
${ }^{5}$ It was proven by Apéry [17] that $\zeta(3)$ is irrational. Then Rivoal [19 proved that one of the nine numbers $\zeta(5), \ldots, \zeta(21)$ is irrational. One of the most recent theorem proved by Zudilin [20] says that one of the four values $\zeta(5), \ldots, \zeta(11)$ is irrational. (See also the paper by D. Zagier [18] and the paper by Yu. Nesterenko [21]).
} 
where

$$
A_{ \pm}=\frac{1 \pm \sqrt{1-4 x}}{2}
$$

In particular when $x \rightarrow 1$ one gets

$$
P(n, 1)=\left\{\begin{array}{rll}
1, & n=6 k \quad \text { or } \quad n=6 k+1 \\
0, & n=3 k+2 & \\
-1, & n=6 k+3 & \text { or } \quad 6 k+4
\end{array}\right.
$$

Note that the alternating zeta values approaches 1 as the argument gos to infinity

$$
\lim _{s \rightarrow \infty} \zeta_{a}(s)=1
$$

Another nice form of the formula (4.13) can be obtained by substitution

$$
x=\frac{1}{4 \cosh ^{2} \alpha}
$$

Then

$$
P\left(n, \frac{1}{4 \cosh ^{2} \alpha}\right)=\frac{\sinh [(n+1) \alpha]}{2^{n}(n+1) \cosh ^{n} \alpha \sinh \alpha}
$$

We see that when $\alpha$ tends to zero

$$
\lim _{\alpha \rightarrow 0} P\left(n, \frac{1}{4 \cosh ^{2} \alpha}\right)=\frac{1}{2^{n}}
$$

Let us remark that this result appeared to coincide with the limiting formula (3.26). We do not know if this is accidental or there is a reason for this.

Let us briefly discuss the generating function for the values $P(n)$

$$
\Psi(y)=\sum_{n=0}^{\infty} y^{n} P(n)
$$

where $P(0)=1$ by definition. Taking into account the conjectures (4.9) and (4.10) we can easily get the two first terms for the generating function $\Psi(y)$

$$
\Psi(y)=-\frac{\ln (1-y)}{y}+\frac{y^{2}}{3(1-y)^{3}} \ln 2+\ldots
$$

As we discussed in [8] and [10] we expect that for $n \gg 1$

$$
P(n) \sim e^{-\kappa n^{2}}
$$

If we substitute it formally into eq. (4.20) with $y=e^{u}$ then we can expect that

$$
\Psi\left(e^{u}\right) \sim \tilde{\Psi}(u)=\sum_{n=0}^{\infty} e^{-\kappa n^{2}+u n}
$$

so that the function $\tilde{\Psi}(u)$ satisfies the functional equation

$$
\tilde{\Psi}(u)+\tilde{\Psi}(-u)-1=\theta_{3}\left(\frac{i u}{2}, e^{-\kappa}\right)
$$


where $\theta_{3}$ is the third Jacobi theta function with the nome $e^{-\kappa}$. So we can expect that the generating function (4.20) may be related with the elliptic functions and have some automorphic properties.

Another possibility is to put into the r.h.s. of eq. (4.20) the values $P(n, x)$ given by (4.13) instead of $P(n)$ then

$$
\Psi(x, y)=\sum_{n=0}^{\infty} y^{n} P(n, x)=\frac{1}{y \sqrt{1-4 x}} \ln \frac{2-y+y \sqrt{1-4 x}}{2-y-y \sqrt{1-4 x}}
$$

Another generating function looks much simpler

$$
\Psi^{\prime}(x, y)=\sum_{n=0}^{\infty}(n+1) y^{n} P(n, x)=\frac{1}{1-y+x y^{2}}
$$

\section{Discussion}

The main point of this communication was to consider the emptiness formation probability in the inhomogeneous case. The basic advantage of the inhomogeneous case is that we have more parameters at our disposal and relation to the qKZ. We derived three general properties of $P_{n}$ from qKZ, which appeared to be extremely useful. From our experience of the direct calculation of $P_{n}$ with $n \leq 4$ we conjectured a general ansatz for $P_{n}(3.20)$. We established that the ansatz (3.20) with the first and the second property from the Section 2 [ namely (2.8)] completely fix the answer. The third property (2.9) turns out to be a corollary. This observation allowed us to evaluate $P_{5}$ and $P_{6}$ very efficiently [ in the inhomogeneous case]. The homogeneous limit of $P_{5}$ reproduced the expression, which we obtained in paper [10] by going through hard and long computations. That time we were not sure that we will be able to calculate $P_{6}$ at all. Now it is possible to do it very quickly just by taking the homogeneous limit of our result in the inhomogeneous case. In the next publications we are planning to prove our general ansatz (3.20) and evaluate $P(n)$ for arbitrary $n$. Actually we think that qKZ approach is so powerful that we will be able to evaluate any correlation function in the Heisenberg XXX model and to show that it has a structure similar to $P(n)$

$$
F\left(z_{1}, \ldots, z_{n}\right)_{\epsilon_{1}, \ldots, \epsilon_{n}}=\sum_{l=0}^{\left[\frac{n}{2}\right]}\left\{A_{n, l}^{\epsilon_{1}, \ldots, \epsilon_{n}} \prod_{j=1}^{l} G\left(z_{2 j-1}-z_{2 j}\right)+\text { permutations }\right\}
$$

where $\epsilon_{j}= \pm 1$

$$
F\left(z_{1}, \ldots, z_{n}\right)_{\epsilon_{1}, \ldots, \epsilon_{n}}=\left\langle\mathrm{GS}\left|\prod_{j=1}^{n} P_{j}^{\epsilon_{j}}\right| \mathrm{GS}\right\rangle
$$

and

$$
P_{j}^{ \pm}=\frac{1 \pm \sigma_{j}^{z}}{2}
$$

We may also expect that similar to the functions $A_{n, l}=A_{n, l}^{+, \ldots,+}$ from $(3.20)$ the functions $A_{n, l}^{\epsilon_{1}, \ldots, \epsilon_{n}}$ are rational of their arguments $z_{1}, \ldots, z_{n}$ also.

\section{Acknowledgements}

The authors would like to thank A. Abanov, R. Flume, M. Jimbo, D. Kreimer, M. Lashkevich, S. Lukyanov, T. Miwa, Yu. Nesterenko, P. Pyatov, V. Tarasov, D. Zagier and A. Zamolodchikov for 
useful discussions. This research has been supported by the following grants: NSF grant PHY9988566, the Russian Foundation of Basic Research under grant \# 01-01-00201, by INTAS under grants \#00-00055 and \# 00-00561. HEB would like to thank the administration of the Max-Planck Institute for Mathematics for hospitality and perfect conditions for the work. HEB and FAS are grateful to organizers of the International Workshop "Conformal Field Theory and Integrable Models", Chernogolovka, September 2002, Russia for opportunity to present this work.

\section{References}

[1] H. Bethe, Zeitschrift für Physik, 76, 205 (1931)

[2] L. Hulthén, Ark. Mat. Astron. Fysik A 26, 1 (1939).

[3] N. Bogoliubov, A.Izergin, V.Korepin Quantum Inverse Scattering Method and Correlation Functions. Cambridge University Press (1993)

[4] M. Jimbo, K. Miki, T. Miwa, A. Nakayashiki, Phys. Lett. A168 (1992) 256-263.

[5] M. Jimbo, T. Miwa ,Algebraic Analysis of Solvable Models. Regional Conference Series in Mathematics, 85, AMS (1995)

[6] M. Jimbo and T. Miwa, "Quantum $K Z$ equation with $|q|=1$ and correlation functions of the XXZ model in the gap-less regime”, J. Phys. A 29 (1996) 2923-2958.

[7] F. Essler, A.G. Izergin, V.E. Korepin, D.B. Uglov, Phys. Lett. A190 (1994) 182-184.

[8] H.E. Boos and V.E. Korepin, "Quantum spin chains and Riemann zeta function with odd arguments", J. Phys.A: Math. Gen 34 (2001) 5311-5316; hep-th/0104008.

[9] H.E. Boos and V.E. Korepin, "Evaluation of integrals representing correlations in XXX Heisenberg spin chain", "Integrable Systems and Beyond", volume "MathPhys Odyssey 2001" in Progress in Mathematics dedicated to the sixties birthday of Professor Barry McCoy, published from Birkhauser, hep-th/0105144.

[10] H.E. Boos, V.E. Korepin, Y. Nishiyama and M. Shiroishi, "Quantum Correlations and Number Theory", to appear in J. Phys.A: Math. Gen, cond-mat/0202346.

[11] V.G. Knizhnik and A.B. Zamolodchikov, "Current algebra and Wess-Zumino model in two dimensions", Nucl. Phys. B247 (1984) 83-103.

[12] I.B. Frenkel and N.Yu. Reshetikhin, Comm. Math. Phys, 146 (1992) 1.

[13] A. Abanov and V.Korepin, cond-mat/0206353.

[14] V.Korepin, S.Lukyanov, Y. Nishiyama and M. Shiroishi, cond-mat/0210140.

[15] F.A.Smirnov, Form Factors in Completely Integrable Models of Quantum Field Theory. Adv. Series in Math. Phys. 14, World Scientific, Singapore (1992) 
[16] F.A. Smirnov, Int. Jour. Math. Phys. (Suppl. 1B), A7 (1992) 813

[17] R. Apéry, "Irrationalité de $\zeta(2)$ et $\zeta(3)$ ”, Astérisque 61 (1979) 11-13.

[18] Don Zagier, "Values of Zeta Functions and their Applications", First European Congress of Mathematics, Vo.II (Paris, 1992) Prog.Math., Birkhauser, Basel-Boston, page 497, 1994

[19] T. Rivoal, C. R. Acad. Sci. Paris Sr. I Math. 331 (2000), no. 4, 267-270 and http://front.math.ucdavis.edu/math.NT/0104221

[20] W. Zudilin, One of the numbers $\zeta(5), \zeta(7), \zeta(9), \zeta(11)$ is irrational", Uspekhi Mat. Nauk [Russian Math. Surveys] 56:4 (2001), 149-150;

"Irrationality of values of Riemann's zeta function", Izv. Ross. Akad. Nauk Ser. Mat. [Russian Acad. Sci. Izv. Math.] 66:3 (2002), 49-102.

[21] Yu.V. Nesterenko, "Integral identities and constructions of approximations to zeta values", Actes des 12èmes rencontres arithmétiques de Lille (July, 2001), J. Théorie Nombres Bordeaux (2002), to appear. 